\title{
Temperature and doping dependence of fluorescence lifetime in Yb:YLF (role of impurities)
}

\author{
Umit Demirbas,,${ }^{1,2,}{ }^{*}$ Jelto Thesinga, ${ }^{1}$ Martin Kellert, ${ }^{1}$ Mikhail Pergament ${ }^{1}$, \\ AND FRANZ X. KÄRTNER ${ }^{1,3,4}$ \\ ${ }^{I}$ Center for Free-Electron Laser Science, Deutsches Elektronen-Synchrotron DESY, Notkestraße 85, 22607 Hamburg, Germany \\ ${ }^{2}$ Laser Technology Laboratory, Department of Electrical and Electronics Engineering, Antalya Bilim University, Antalya, Turkey \\ ${ }^{3}$ Physics Department, University of Hamburg, Luruper Chaussee 149, 22761 Hamburg, Germany \\ ${ }^{4}$ The Hamburg Centre for Ultrafast Imaging, Luruper Chaussee 149, 22761 Hamburg, Germany \\ *uemit.demirbas@cfel.de
}

\begin{abstract}
We have investigated temperature and doping dependence of fluorescence lifetime in Yb:YLF crystals in the 78-300 $\mathrm{K}$ range, for samples with Yb-doping levels of $0.5 \%, 1 \%$ and $25 \%$. Radiation-trapping prolonged fluorescence lifetimes are first measured to understand the pros and cons of this inevitable process on fractional thermal load and laser gain. Later, pinhole method was used to acquire ideally trapping free fluoresce lifetimes. For the lowly Yb-doped samples, fluorescence lifetime was measured to be $1.97 \mathrm{~ms}$ and $2.1 \mathrm{~ms}$ at $78 \mathrm{~K}$ and $300 \mathrm{~K}$, respectively. For the $25 \% \mathrm{Yb}$-doped sample, strong upconversion emission due to the presence of Er, Tm and Ho impurities were observed. A doubleexponential decay behavior, combined with excitation intensity dependent decay profile was also revealed for this highly doped sample. Even with the pinhole method, the $300 \mathrm{~K}$ fluorescence lifetimes was measured as $4.5 \mathrm{~ms}$, showing the difficulty to acquire radiation-trapping free signals at high doping levels. Time dynamics of upconverted emission in different spectral regions were also recorded, which provides hints on the energy transfer mechanisms between the Yb ion and the other rare-earth impurities.
\end{abstract}

Keywords: Solid-state lasers; laser crystals; cryogenic lasers; $\mathrm{Yb}^{+3}$-doped $\mathrm{YLiF}_{4}$; fluorescence lifetime.

\section{Introduction}

Yb:YLF (Yb:LiYF4) is an interesting material that attracts the attention of optics community, not only as a broadband near infrared laser/amplifier gain medium [1-11], but also as a solid-state optical refrigerating medium [12-18]. From the laser scientist point of view, operating Yb:YLF at cryogenic temperatures enables increased gain and better thermal management, and provides laser sources with 2-3 orders of magnitude higher average power in comparison with roomtemperature operation [19-28]. For the optical refrigeration community, solid-state cooling to cryogenic temperatures using Yb:YLF is an important tool to develop nanometer scale quantum optical devices [16]. The Yb-doping of YLF is an important parameter that should be carefully optimized for achieving the desired laser performance [9], and high doping levels are usually required for laser geometries such as thindisks or slabs [3]. Compared to the other well-known laser materials like Ti:Sapphire and Yb:YAG, Yb:YLF is more forgiving at high doping levels. Relatively efficient lasing/amplification have already been obtained using 20$60 \%$ Yb-doped YLF samples [1-5, 9, 20, 29]; however, most of the time at the expense of deleterious thermal effects, which usually requires quasi continuous-wave operation or amplification at low repetition rates. For optical cooling applications based on anti-Stokes emission of Yb:YLF crystals, one ideally needs very high doping levels to achieve efficient absorption near $\sim 1020 \mathrm{~nm}$ (this line lies at the long wavelength edge of the absorption spectrum). On the other hand, efficient cooling of the crystal also requires samples with minimal impurities and minimal parasitic background absorption $[15,17,18]$, and so far the optimum doping level stays around $10 \%$. In short, for laser and optical refrigeration communities, it is important to understand the variation of thermo-opto-mechanical parameters of Yb:YLF with doping and temperature $[9,21,30,31]$.

In this work, we have focused our attention on fluorescence lifetime, which is an important parameter for the design of efficient laser/amplifier systems. Fluorescence lifetime could also provide information on the impurities of the crystal, and hence it is potentially important for understanding the optical cooling performance of the crystal. As is well summarized in [32], Yb:YLF has a simple energy level structure, and high-purity samples ideally do not possess excited-state absorption, cross-relaxation, or up-conversion that could all effect the fluorescence lifetime of the laser active level (see Fig. 1 in [32]). On the other hand, the crystal 
field is quite weak in YLF, and as a result the absorption and emission spectra strongly overlap, creating radiation trapping effects that could significantly influence the measured fluorescence lifetime [32-40]. The radiation trapping mechanism, which is also known as radiative diffusion, is crystal geometry dependent due to presence of effects such as total internal reflection [40] and is not easy to model accurately [38]. The process involves reabsorption of the emitted fluorescence, creates an additional heat load on the material beyond the quantum defect [41-43], and hence is not desired in terms of thermal behavior. On the other hand, it can be also beneficial for laser amplifiers: enables storage of inversion on a longer timescale than radiative lifetime and facilitates higher small single pass gain (if it is reabsorbed in a region that overlaps with the seed). As a side note, a redistribution of inverted ion population might also occur via nonradiative energy transfer process due to electrical interactions such as dipole-dipole activity [38, 44].

Besides radiation trapping, Boulon et al. has already shown that there are radiation quenching effects in highly-Ybdoped Yb:YLF samples [32], where lifetime is shortened by energy diffusion towards traps (quenching centers). These radiation traps are not always well-known [38]: but in the case of $Y b: Y L F$, existence of trace amounts of rare earth impurities (e.g. Er, Tm, Ho) is shown to create radiation quenching channels $[17,32,45]$. The energy transfer from the excited $\mathrm{Yb}$ ion into these impurities creates a loss of useful inversion, and results in concentration quenching of the fluorescence lifetime [15]. At elevated temperatures multiphonon relaxation may also create a nonradiative relaxation channel[46], but the YLF lattice is known to be resistant towards this process [47]. Cooperative emission of neighboring $\mathrm{Yb}$ ions (pair emission) is also known to create green emission in the 490-510 $\mathrm{nm}$ range in Yb:YLF samples [32], but this process has been shown to be quite weak in Yb:YLF. Furthermore, amplified spontaneous emission (ASE) can also play a role in the population dynamics of highly-doped Yb:YLF crystals [48, 49]. As we tried to summarize shortly, despite the simple looking energy structure, the emission dynamics could be rather complex in Yb-doped gain media as well. Unfortunately, despite the importance of the fluorescence lifetime for the design of efficient laser/amplifier systems, to our knowledge, it is so far only investigated in a few studies for Yb:YLF $[31,32,50]$.

In this work, to extend the knowledge on this topic, we have investigated the variation of fluorescence lifetime of $\mathrm{Yb}$ :YLF with temperature in the $78-300 \mathrm{~K}$ range using crystals with doping levels of $0.5 \%, 1 \%$ and $25 \%$. Our initial experiments show the role of temperature and doping in increasing the effective fluorescence lifetime of $\mathrm{Yb}$ :YLF crystals via radiation trapping. Later also the pinhole method was used to ideally measure radiation-trapping free intrinsic fluorescence lifetimes, where good agreement with literature was observed for the lowly $\mathrm{Yb}$-doped crystals $(1.97 \mathrm{~ms}$ at 78 $\mathrm{K}$ and $2.1 \mathrm{~ms}$ at $300 \mathrm{~K}$ ). For the $25 \% \mathrm{Yb}$-doped YLF sample, radiation trapping effects were very strong and the measured room-temperature fluorescence lifetime was as high as $4.6 \mathrm{~ms}$ even when using the pinhole method. We have also identified the presence of Er, Tm and Ho impurities in this highly doped crystal by observing a strong upconversion spectrum in the visible range. Moreover, fluorescence decay curves with double exponential behavior and excitation intensity dependent fluorescence lifetime behavior were also observed in the 25\% Yb-doped YLF sample. The measured intensity dependent behavior, which to our knowledge, reported to the first time in Yb:YLF, might be important for the design of efficient laser amplifier systems. We have further measured the temporal dynamics of the fluorescence emission at different spectral regions of the upconversion spectrum and acquired hints on the energy transfer mechanisms between the rare earth impurities.

The paper is organized as follows: In Section 2, we summarize the experimental setup and the methodology. In Section 3, we present the experimental results in detail, and in Section 4 we close with a brief discussion.

\section{Experimental}

Several Yb:YLF crystals with Yb-doping of $0.5 \%, 1 \%$ and $25 \%$ were available for the experiments. The $0.5 \%$ and $1 \%$ doped crystals were 2-cm long, and had an aperture of $10 \mathrm{~mm}$ by $15 \mathrm{~mm}$. The $25 \%$ Yb-doped crystals were $1.75 \mathrm{~mm}$ long and had an aperture of $6 \mathrm{~mm}$ by $8 \mathrm{~mm}$. The front and back surfaces of all samples were anti-reflection coated for $1 \mu \mathrm{m}$ region, which minimizes trapping of radiation via total internal reflection process. A fiber-coupled, $960 \mathrm{~nm}$ laser diode was used as the excitation source. The pump light from the fiber output is collimated and imaged inside the gain medium using a set of appropriate lenses, and a flattop beam profile with a beam diameter of around $2 \mathrm{~mm}$ was obtained on the crystals. In typical emission lifetime measurements, Yb:YLF crystals are excited with $200 \mu$ s long pump pulses with $2 \mathrm{~kW}$ of peak power at repetition rates between 0.1 to 1 $\mathrm{Hz}$ (a low rep rate is used in highly doped crystal). Despite the large peak power, due to the low duty cycle employed, the average absorbed pump power was below $0.5 \mathrm{~W}$ in these measurements, and hence the heat load on the crystals was rather low. For measuring the excitation intensity dependence of the fluorescence lifetime, a $2 \mathrm{~ms}$ pump pulse was used and the peak power of the pulses has been varied to adjust the incident excitation energy level on the crystals. With that, a maximum incident energy of $4 \mathrm{~J}(2 \mathrm{~kW}$ for $2 \mathrm{~ms})$ was available for the experiments, which corresponds to a fluence of $125 \mathrm{~mJ} / \mathrm{cm}^{2}$.

Fig. 1 shows a simple schematic of the fluorescence lifetime measurement setup. The fluorescence decay signal was measured at $90^{\circ}$ with respect to the direction of excitation beam. A $1000 \mathrm{~nm}$ high-pass filter (Thorlabs, FELH1000) was also implemented to cut out any remaining scattered pump signal (which was already at a low level due to usage of high quality crystals and measurement at $90^{\circ}$ ). The time-dependent fluorescence decay signal was monitored using a free-space $350 \mathrm{MHz}$ Si detector (Thorlabs, DET10A) which possesses a spectral response in the 200-1100 $\mathrm{nm}$ range. The signal was recorded with a $600 \mathrm{MHz}$ digital sampling oscilloscope, where a variable resistance terminator was used to adjust the 
signal strength on the oscilloscope. The system impulse response was measured to be less than $20 \mu$ s even with the largest termination resistance $(50 \mathrm{kohm})$ used in this study. Pinholes with diameters between $10 \mu \mathrm{m}$ and $2 \mathrm{~mm}$ were used for the estimation of radiation-trapping free lifetimes. The high-pass optical filter in front of the detector was replaced by different band-bass and low-pass filters for the measurement of time dynamics of the upconversion spectra in different spectral regions (filters FES0900, FGB37, FB800-40, FB48010, FB500-40 from Thorlabs).

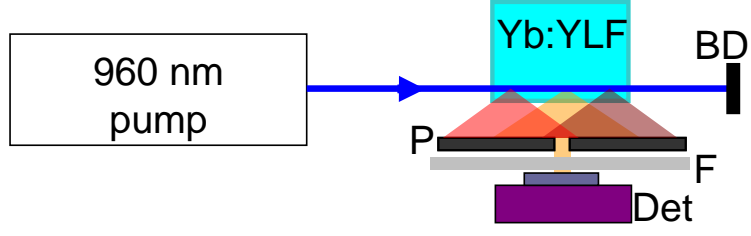

Figure 1: A simplified schematic of the fluorescence lifetime measurement setup. The simple system consists of a pulsed high power $960 \mathrm{~nm}$ diode $(200 \mu \mathrm{s}$ long pulses with $2 \mathrm{~kW}$ peak power) that is used as the excitation source, Yb:YLF crystals with different Ybdoping levels, and a fast $\mathrm{Si}$ detector oriented at 90 degrees to the pump line for background-free lifetime measurements. P: Pinhole, F:Filter, BD: beam dump, Det: Detector.

For temperature controlled experiments, the crystals were indium bonded from the top side to a multi-stage pyramidal cold head, which was cooled to cryogenic temperatures by boiling liquid nitrogen. Several silicon-diode based thermal sensors connected to the cold head provided real time temperature information of the cold head with $\pm 0.1 \mathrm{~K}$ accuracy. For temperature dependent fluorescence lifetime measurements, the slow (6-7 hours) dewar cooling cycle is used to acquire the data, as was also employed and described in detail in [51]. During the slow cooling process, the crystals and the cold head are in thermal equilibrium, and the crystal temperature can be accurately measured using the thermal sensors.

In the upconversion emission measurements, the emission spectra were again measured at $90^{\circ}$ angle to the pump propagation direction using an Ocean Optics spectrometer with a spectral resolution of $0.5 \mathrm{~nm}$ in the $200-1100 \mathrm{~nm}$ range. The measured signal is corrected for the overall spectral response of the system (CCD array and grating). The $\mathrm{Ho}^{+3}$ ion emission near the $2 \mu \mathrm{m}$ region is recorded using a $5 \mathrm{~nm}$ resolution NIR Quest spectrometer.

\section{Fluorescence lifetime measurement results}

The results for the fluorescence lifetime measurements are presented in several sub-sections. In Section 3.1, we outline the effect of temperature on lifetime, whereas in Section 3.2 we discuss the results obtained with the pinhole method. Variation of the measured temporal emission dynamics with excitation energy will be elaborated in Section 3.3. Finally, in Sections 3.4 and 3.5, we present the measured spectral and time domain picture for the upconversion mechanism.

\subsection{Temperature dependence of fluorescence lifetime}
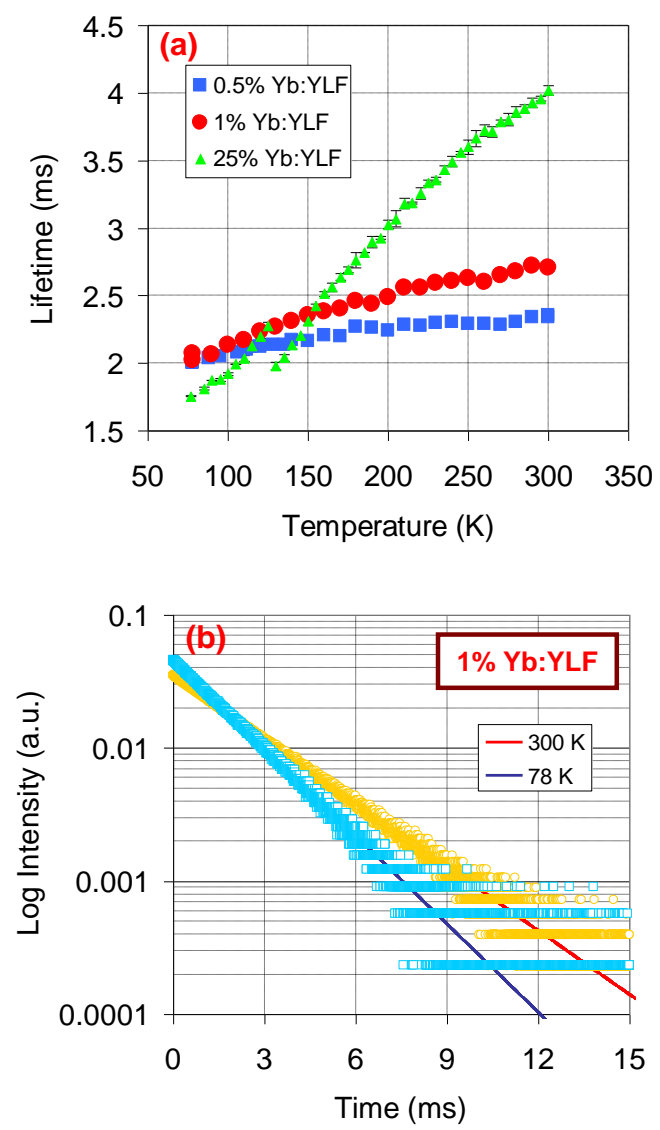

Figure 2: (a) Measured variation of fluorescence lifetime with temperature in the $78-300 \mathrm{~K}$ range, for $0.5 \%, 1 \%$ and $25 \% \mathrm{Yb}$-doped YLF samples. The data is taken without using a pinhole, and at incident excitation energy of around $400 \mathrm{~mJ}(200 \mu \mathrm{s}$ long pulses with $2 \mathrm{~kW}$ peak power). The results are obtained via making a single exponential fit to the measured decay curves. (b) Sample decay curves and single exponential fit to the data for the $1 \% \mathrm{Yb}: \mathrm{YLF}$ sample. Note that, the limited dynamic range of the measurement system creates the observed noise for signal levels below $\sim 0.001$.

We start by presenting our results in Fig.2 (a), which shows the measured temperature dependence of fluorescence lifetime of Yb:YLF gain medium in the 78-300 $\mathrm{K}$ range. The measurements were performed at three different $\mathrm{Yb}$-doping levels: $0.5 \%, 1 \%$ and $25 \%$. The data in Fig. 1 (a) is obtained by making a single exponential fit to the measured fluorescence decay curves, in the form:

$$
F(t)=C \operatorname{Exp}\left(-\frac{t}{\tau_{0}}\right) .
$$

Above, $\mathrm{F}(\mathrm{t})$ is the recorded/measured time-dependent decay curve, $\mathrm{t}$ is time, $\mathrm{C}$ is the amplitude (arbitrary constant), and $\tau_{0}$ is the fluorescence lifetime of the system that is found by minimizing the sum of the squares of the differences between the measured and predicted decay (known as the method of least squares). For the lowly Yb-doped samples $(0.5 \%$ and $1 \%$ ), a single exponential fit matched the experimentally measured decay curves very well. As an example, Fig. 2(b) shows sample decay curves measured for $1 \% \mathrm{Yb}$-doped YLF sample taken at temperatures of $78 \mathrm{~K}$ and $300 \mathrm{~K}$. As an 
exception, for the $25 \%$ doped sample, we have noticed that the measured decay curve is better matched by a double exponential decay behavior, and this issue will be discussed using Fig. 3 a little later.

We see from Fig. 2 (a) that, for the lowly Yb-doped samples, the measured fluorescence lifetime decreases slowly and steadily with decreasing temperature, and has a measured value of around $2.05 \mathrm{~ms}$ at $78 \mathrm{~K}(2.07 \mathrm{~ms}$ for $1 \%$ and $2.02 \mathrm{~ms}$ for $0.5 \%$ ). Note that, the data in Fig. 2 is taken without using the pinhole method that minimizes radiation trapping. Hence, we believe that, the increase of measured fluorescence lifetime with temperature is due to the increasing role of radiation trapping: at higher temperatures the absorption and emission bands overlap more and more, increasing the rate of radiation trapping. Also, as the Yb-doping of the sample increases from $0.5 \%$ to $1 \%$, the room-temperature $(\sim 300 \mathrm{~K})$ fluorescence lifetime increased from around $2.35 \mathrm{~ms}$ to $2.7 \mathrm{~ms}$ (due to the increased role of radiation trapping with doping).

As we will present later, the effect of fluorescence trapping could be minimized by use of aperture method, and fluorescence lifetimes that are closer to the radiative lifetime of the material could be measured (Section 3.2). On one hand, the data in Fig. 2 (a) represents a case close to what we have in laser experiments. It shows that, due to radiation trapping, the emitted photons from the material have a non-ignorable chance of reabsorption, and the rate of reabsorption (radiation trapping) increases with temperature and doping. Of course, as a benefit, the radiation trapping process could be advantageous for laser gain especially for laser amplification studies at low repetition rates where one can pump the system longer than the radiative lifetime, and still improve the stored inversion level and hence gain (e.g. a 25\% doped YLF was employed in the $10 \mathrm{~Hz}$ regenerative amplifier of [29], and this sample we have seen increased gain for pump pulsewidths as along as $10 \mathrm{~ms}$ ). On the other hand, each reabsorption event increases the fractional thermal load of the system and for $\mathrm{cw}$ operation or higher repetition rate amplification; the additional heat load might be detrimental for laser/amplifier performance. We believe that, the data in Fig. 2 (a) could be used to estimate the increase in fractional thermal load and gain with temperature and doping, and could be beneficial for modeling of inversion and thermal load in Yb:YLF system.

In Fig. 2 (a), we have seen a rather interesting behavior with the fluorescence lifetime data of the $25 \% \mathrm{Yb}$-doped YLF sample. First of all, the single exponential fit to the measured fluorescence lifetime has a value of $1.76 \mathrm{~ms}$ at $78 \mathrm{~K}$, interestingly a value lower than the known radiative lifetime of the material $(\sim 2 \mathrm{~ms})$. Moreover, there is a sudden drop in the lifetime data at $130 \mathrm{~K}$. To understand this behavior we returned back to our experimental findings more carefully, and realized that at $130 \mathrm{~K}$ we increased the incident excitation energy of the sample from around $400 \mathrm{~mJ}$ to $600 \mathrm{~mJ}$ to improve the measured fluorescence signal strength. It is clear from the results that this clearly changed the trend: the measured fluorescence decay curve shape is excitation energy dependent. As mentioned earlier in the subsection, we have further noticed that, a single exponential does not provide a very good fit to the measured data, and hence we have also tried a double exponential fit to the measured decay:

$$
F(t)=A \operatorname{Exp}\left(-\frac{t}{\tau_{s}}\right)+B \operatorname{Exp}\left(-\frac{t}{\tau_{f}}\right) .
$$

In Equation (2), $\tau_{\mathrm{f}}$ and $\tau_{\mathrm{s}}$ are fast and slow decay constants of the system, and $\mathrm{A}$ and $\mathrm{B}$ are their corresponding amplitudes, and other parameters are as defined earlier.

The double exponential fit to the measured decay curves of the $25 \%$ Yb-doped YLF sample are summarized in Fig. 3 (the fit searches for values of $\mathrm{A}, \mathrm{B}, \tau_{\mathrm{f}}$ and $\tau_{\mathrm{s}}$ that minimizes the sum of the squares of the differences between the measured data and predicted double-exponential decay). Sample experimentally measured decay curves and double exponential fit to the experimental data is shown in Fig. 3 (c). For each temperature we had at least 3 experimentally measured decay curves (similar to the ones shown in Fig. 3 (c)) and the reported decay constant values in Fig. 3 (a) are average values and the error bars in the graph show the standard deviation of the data. Besides showing the best-fit values for fast $\left(\tau_{\mathrm{f}}\right)$ and slow $\left(\tau_{\mathrm{s}}\right)$ decay constants obtained using Eq. (2), Fig. 3 (a) also shows the single-exponential fit result $\left(\tau_{0}\right)$ acquired using Eq. (1). Furthermore, Fig. 3 (b) shows the ratio of slow decay magnitude (A) to fast decay magnitude (B). We clearly see from Fig. 3 (a) that, the values of single-exponential decay constant $\left(\tau_{0}\right)$, as well as the slow $\left(\tau_{\mathrm{s}}\right)$ ) and fast $\left(\tau_{\mathrm{f}}\right)$ components of double-exponential fit increase with increasing temperature. This again we believe is due of the increased role of radiation trapping with temperature.

As we see from Fig. 3 (b), the strength/magnitude of the slow component is 3-6 times higher than the fast component (A/B ratio in Eq. 2). Note also from Fig. 2 (b) that, at $130 \mathrm{~K}$, when the pump excitation intensity is increased, the fast component get stronger (so the ratio of slow/fast decay drops). As a result of increased strength of fast component, the lifetime obtained via a single exponential fit $\left(\tau_{0}\right)$ decreases and creates the sharp drop we noticed in Fig. 2 (a).

We believe that the slow component of the decay curve $\left(\tau_{\mathrm{s}}\right)$ corresponds to the main lifetime of the upper laser level. The slow component has a measured value of $2.15 \mathrm{~ms}$ at 78 $\mathrm{K}$, which increases to around $4.5 \mathrm{~ms}$ at $300 \mathrm{~K}$. We further believe that this increase is due to the presence of strong radiation trapping in the $25 \%$ doped sample. The fast component $\left(\tau_{\mathrm{f}}\right)$ has a measured value of $0.5 \mathrm{~ms}$ at $78 \mathrm{~K}$, which increases to around $1.5 \mathrm{~ms}$ at $300 \mathrm{~K}$. Note from Fig. 3 (b) that, the fast component loses strength as the temperature of the sample increases, and we think this is due the decrease of absorption with temperature (or due to decreased inversion of the sample with temperature). 

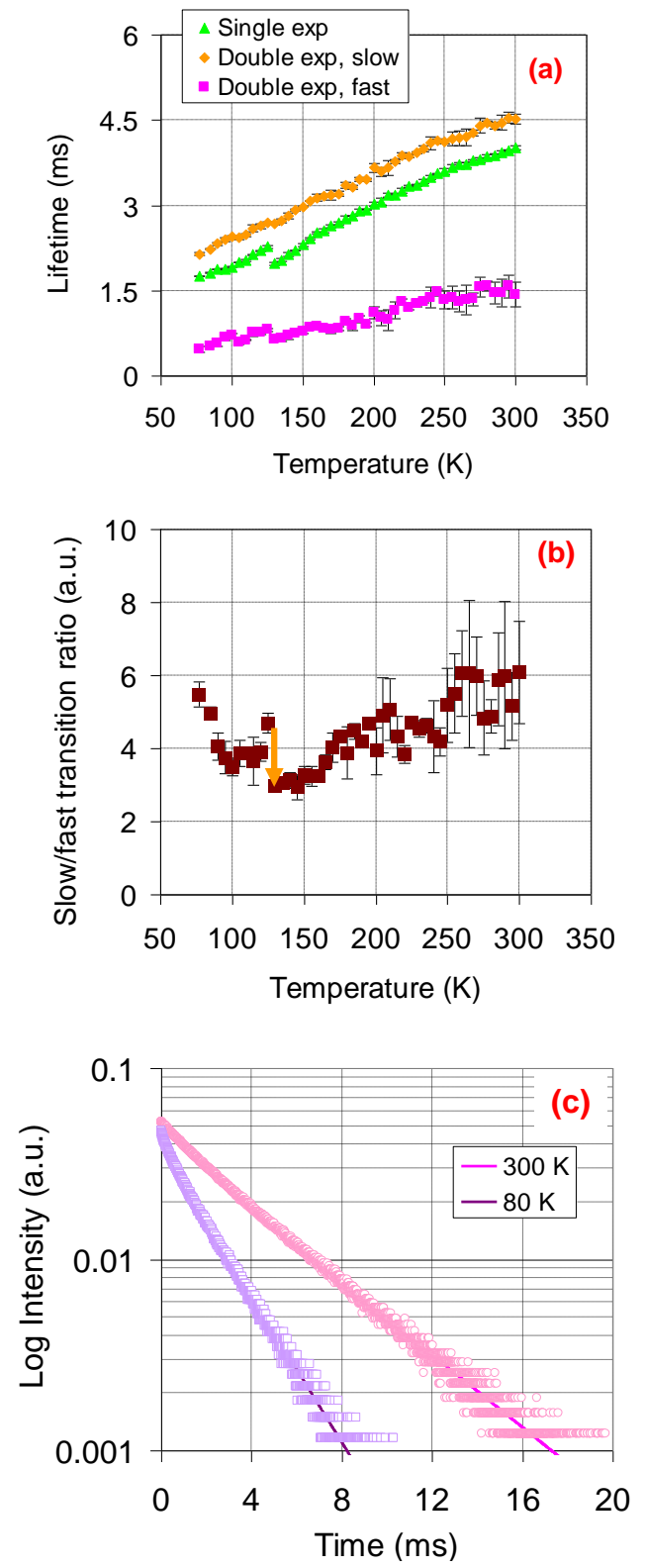

Figure 3: (a) Measured variation of fluorescence lifetime with temperature for the $25 \% \mathrm{Yb}$-doped YLF sample. Results for single $\left(\tau_{0}\right)$, and double exponential fit are shown. Double exponential fit results in a slow $\left(\tau_{\mathrm{s}}\right)$ and a fast $\left(\tau_{\mathrm{f}}\right)$ component. (b) Corresponding variation of the ratio of the slow and fast component strength with temperature for the double exponential fit is shown (A/B ratio in Eq. (2)). The arrow indicates the jump due to increased excitation fluency (from $400 \mathrm{~mJ}$ to $600 \mathrm{~mJ}$ ). (c) Sample double exponential fit to the experimental data at $80 \mathrm{~K}$ and $300 \mathrm{~K}$.

We could not find any discussion in the literature related to double exponential decay behavior in Yb:YLF, but we suspect that this fast process $\left(\tau_{\mathrm{f}}\right)$ is due to the high level of inversion in the sample created by high energy excitation pulse used in our study (pump settings are typical values used in amplifiers). In our view, the observed initial fast decay might be: (i) due to the redistribution of energy from excited $\mathrm{Yb}^{+3}$ ions to other rare earth impurities via upconversion, and/or (ii) due to the amplified spontaneous emission process, as we will discuss further in the coming sections [48, 49].

\subsection{Minimizing radiation trapping}

In the earlier section, we have presented measured variation of fluorescence lifetimes with temperature, but the data was taken without care for radiation trapping. In this section, we will present fluorescence lifetime measurements at $78 \mathrm{~K}$ and $300 \mathrm{~K}$ where we tried to measure the bare fluorescence lifetimes with minimal contribution from radiation trapping. For that, as described in detail in [52-54], we have looked at the variation of measured fluorescence lifetime with the pinhole size used in measurements.
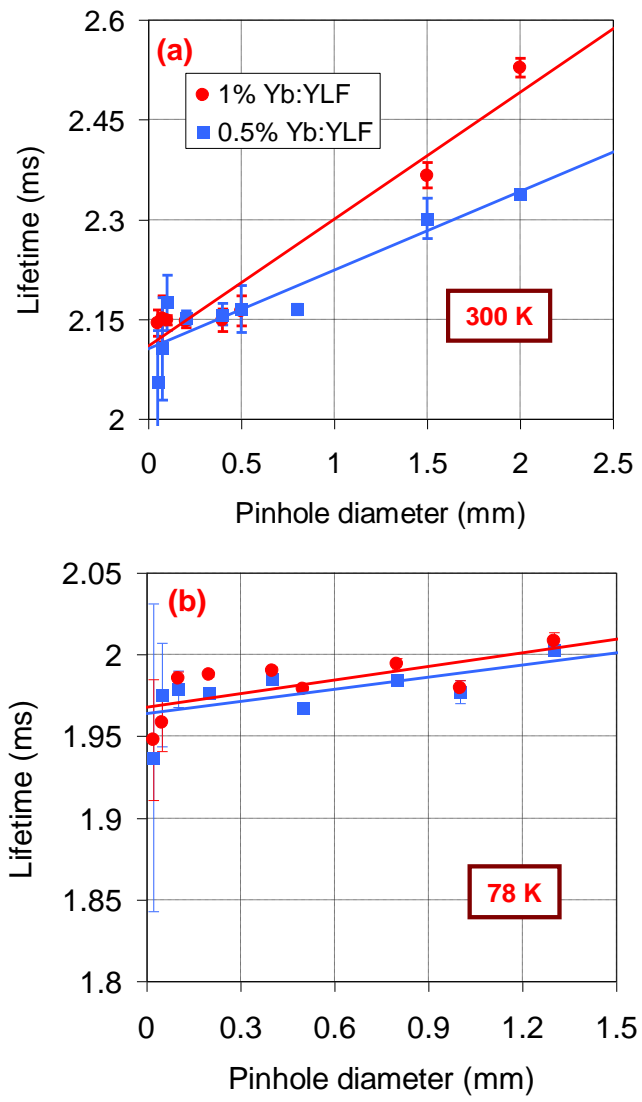

Figure 4: Measured variation of fluorescence lifetime with circular pinhole aperture diameter for the $0.5 \%$ and $1 \%$ Yb-doped YLF samples at (a) $300 \mathrm{~K}$ and (b) $78 \mathrm{~K}$. The reduced pinhole diameter minimizes radiation trapping effects. The data is taken at incident excitation energy of around $400 \mathrm{~mJ}$, and the results are obtained via making a single exponential fit to the measured decay curves.

Figure 4 shows the dependence of measured fluorescence lifetime to pinhole size for the $0.5 \%$ and $1 \%$ doped YLF samples at 78 and $300 \mathrm{~K}$. For both doping levels, a linear fit to the data shows that, the bare (ideally radiation trapping free) fluorescence lifetime of the samples at $78 \mathrm{~K}$ and $300 \mathrm{~K}$ is around $1.97 \mathrm{~ms}$ and $2.1 \mathrm{~ms}$, respectively. These values are in very good agreement with the values reported by T. Y. Fan et al. for a 5\% Yb-doped YLF sample: $1.99 \mathrm{~ms}$ at $80 \mathrm{~K}$, and $2.08 \mathrm{~ms}$ at $296 \mathrm{~K}$ [31]. Recently, similar values are also 
presented by Korner at al. for a 10-\% doped YLF sample [50]. In our opinion, the slight increase of fluorescence lifetime with temperature from $1.97 \mathrm{~ms}$ to $2.1 \mathrm{~ms}$ might still be due to remaining radiation trapping effects at higher temperatures. We also don't know the role of non-radiative processes in $\mathrm{Yb}$ doped YLF. Hence, it is not clear if these values can be interpreted as radiative lifetime values. However, radiative lifetime values could also be calculated/estimated using the measured absorption/emission spectrum on the basics of reciprocity method, and reported/calculated values in literature are $2.27 \mathrm{~ms}$ [30] and $2.04 \pm 0.18 \mathrm{~ms} \mathrm{[50].} \mathrm{As} \mathrm{a} \mathrm{result,}$ we see that, within experimental error bars, the measured fluorescence lifetime of the samples with pinholes are close to the calculated radiative lifetimes and can potentially be interpreted as radiative lifetime of the $\mathrm{Yb}^{+3}$ ions in the upper laser level.
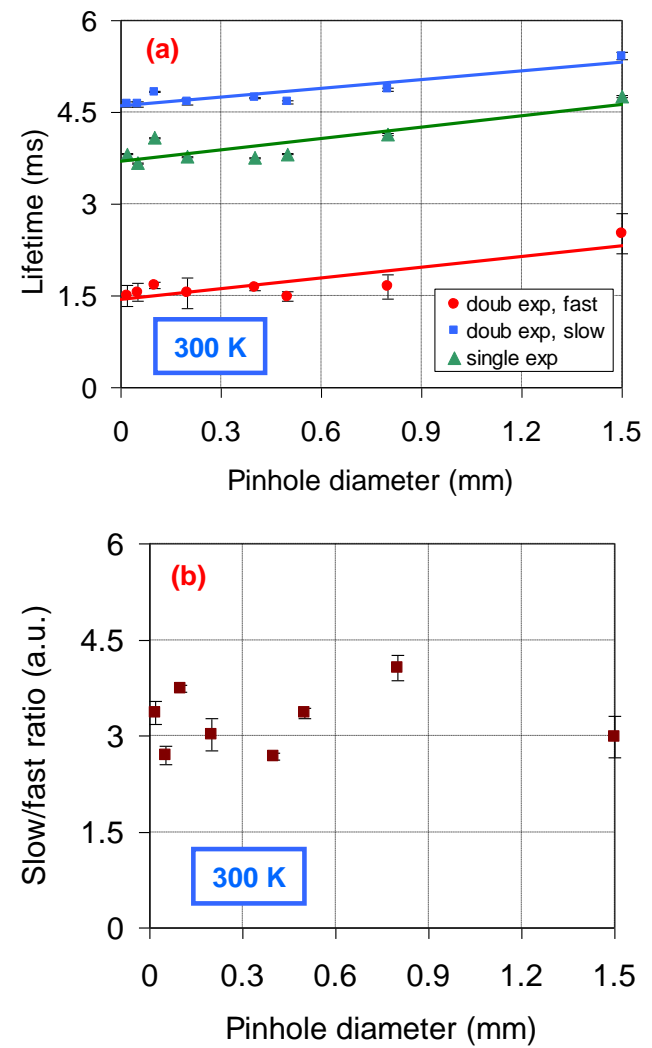

Figure 5: (a) Measured variation of fluorescence lifetime with circular pinhole aperture diameter for the $25 \%$ Yb-doped YLF sample at $300 \mathrm{~K}$. The reduced pinhole diameter minimizes radiation trapping effects. The data is taken at incident excitation energy of around $400 \mathrm{~mJ}$, and the results are obtained via making a double exponential fit to the measured decay curves. (b) Corresponding variation of the ratio of slow and fast component strength of double exponential fit with temperature (A/B ratio in Eq. (2)).

We have also measured the variation of fluorescence lifetime with pinhole diameter for the $25 \%$ doped YLF sample at $78 \mathrm{~K}$ and $300 \mathrm{~K}$. Fig. 5 and Fig. 6 summarizes the results of single and double exponential fit to the measured data. The $300 \mathrm{~K}$ data, which is measured at excitation energy of $400 \mathrm{~mJ}$ shows that with the smaller pinhole size, the measured lifetime decreases in both fast and slow components. The ratio of fast and slow component strengths/magnitudes stays roughly constant, and do not vary with pinhole diameter. The slow and strong component, which we can attribute to the main upper laser level has a lifetime value of around $4.6 \mathrm{~ms}$, which is quite long compared to the low Yb-doped samples. This might be due to the difficulty of getting a completely radiating trapping free measurement in the $25 \% \mathrm{Yb}$-doped sample at room-temperature. As another explanation, other rare earth impurity ions such as $\mathrm{Er}$, Tm and Ho with their metastable levels with long lifetimes might also be acting as reservoirs for the $\mathrm{Yb}$-ion (via energy transfer between the ions), effectively increasing the fluorescence lifetime of the system. However, as we will present below, the $25 \%$ doped crystal does not possess a long fluorescence lifetime at $78 \mathrm{~K}$, and hence we believe that the long fluorescence lifetime measured at $300 \mathrm{~K}$ is most probably due to the inevitable radiation trapping process in this highly doped sample.
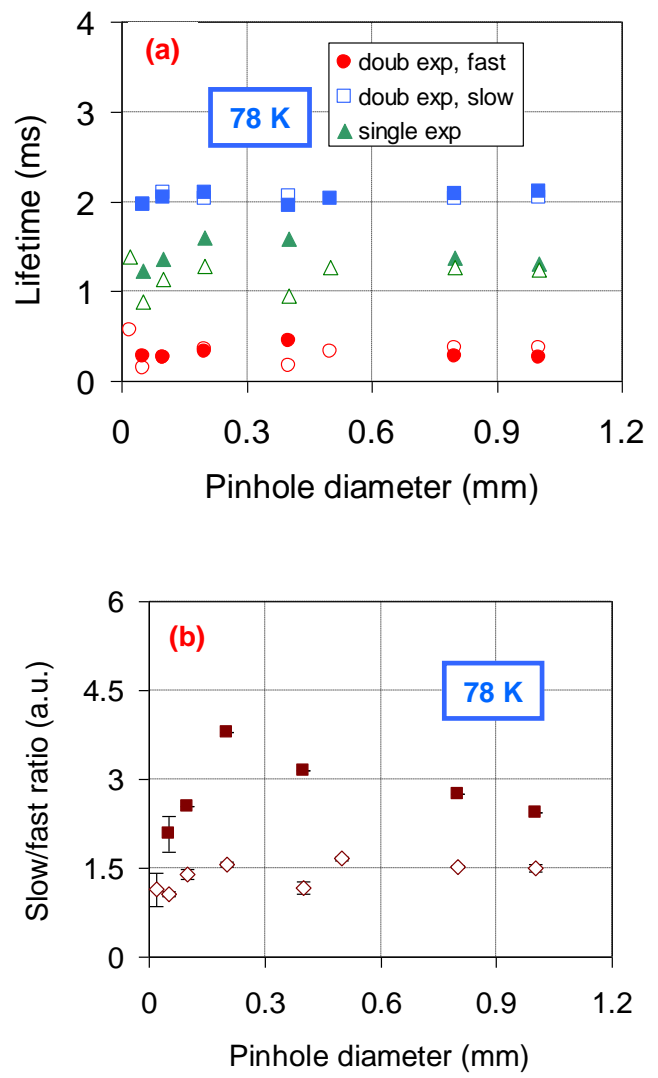

Figure 6: (a) Measured variation of fluorescence lifetime with circular pinhole aperture diameter for the $25 \%$ Yb-doped YLF sample at $78 \mathrm{~K}$. The reduced pinhole diameter minimizes radiation trapping effects. (b) Corresponding variation of the ratio of slow and fast component strength of double exponential fit with temperature (A/B ratio in Eq. (2). The data is taken at incident excitation energies of around $400 \mathrm{~mJ}$ and $4 \mathrm{~J}$ (open markers belong to $4 \mathrm{~J}$ measurement). The results are obtained via making a double exponential fit to the measured decay curves.

At $78 \mathrm{~K}$, we have made two different measurements, one at an incident energy of $400 \mathrm{~mJ}$, and another at an incident 
energy of $4 \mathrm{~J}$ (higher energy measurements are shown with open markers in Fig. 6). Within experimental errors, we did not observe any significant difference on the slow component lifetime of the transition at these different excitation intensities, which remained around $2 \mathrm{~ms}$ in the whole measurement range (Fig. 6 (a)). This clearly shows that at 78 $\mathrm{K}$, the absorption and emission bands of Yb:YLF are well separated, and radiation trapping effects are not strong even for highly doped samples. The measured $2 \mathrm{~ms}$ lifetime is also in agreement with the earlier measurements with the lowly Yb-doped YLF samples. Note that, compared to the $300 \mathrm{~K}$ measurement, the fast component is stronger, and had a lifetime of around $300 \mu \mathrm{s}$. Moreover, within experimental errors, the fast component lifetime $\left(\tau_{\mathrm{f}}\right)$ is similar for both 400 $\mathrm{mJ}$ and $4 \mathrm{~J}$ excitation. Contrarily, as we can see from Fig. 6 (b), the increased excitation intensity clearly increases the strength of the fast decay (A/B ratio in Eq. (2)), as it is also observed in earlier measurements.

\subsection{Intensity dependence of fluorescence lifetime in the $25 \% \mathrm{Yb}$ - doped YLF sample}
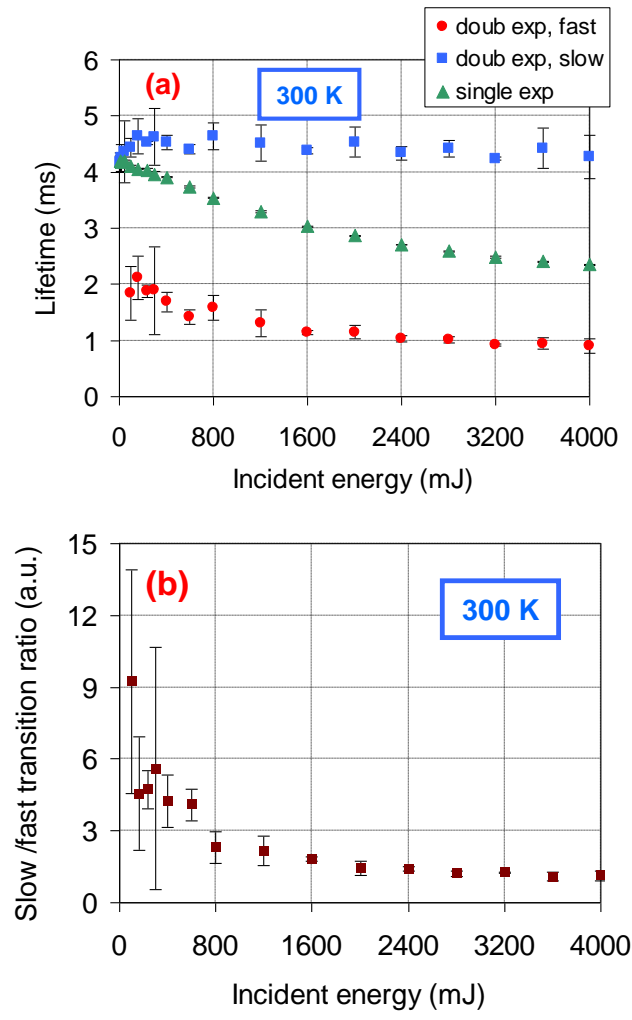

Figure 7: (a) Measured variation of fluorescence lifetime with incident pump excitation energy for the $25 \%$ Yb-doped YLF sample at $300 \mathrm{~K}$. The results are obtained via making a double exponential fit to the measured decay curves. (b) Corresponding variation of the ratio of slow and fast component strength of double exponential fit with temperature (A/B ratio in Eq. (2)). The data is taken using a 50 $\mu \mathrm{m}$ pinhole aperture to minimize radiation trapping effects.

In the earlier sections, we have seen that, for the $25 \% \mathrm{Yb}$ doped sample, the measured fluorescence lifetime decay dynamics shows pump excitation intensity dependence. To investigate this issue further, we have measured the variation of fluorescence lifetime of the 25\% doped YLF sample with excitation intensity at temperatures of $78 \mathrm{~K}$ and $300 \mathrm{~K}$. A 50 $\mu \mathrm{m}$ diameter circular pinhole was used in the measurements to minimize radiation trapping effects.

Fig. 7 summarizes the excitation intensity dependent lifetime measurements at $300 \mathrm{~K}$. The emission decay curves were fitted using single and double exponential assumptions. At low excitation intensities (e.g $100 \mathrm{~mJ}$, see Fig 8 (a)), a single exponential fit provided a very good match to the measured decay. A double exponential fit could also be used, but the strength of additional fast component was rather low (less than $1 / 10^{\text {th }}$ of the slow decay). On the other hand, at high excitation intensities, we have clearly observed a double exponential behavior, and a single exponential assumption was unable to fit to the experimentally measured decay curve (e.g. $4 \mathrm{~J}$ curve in Fig. 8 (b)).
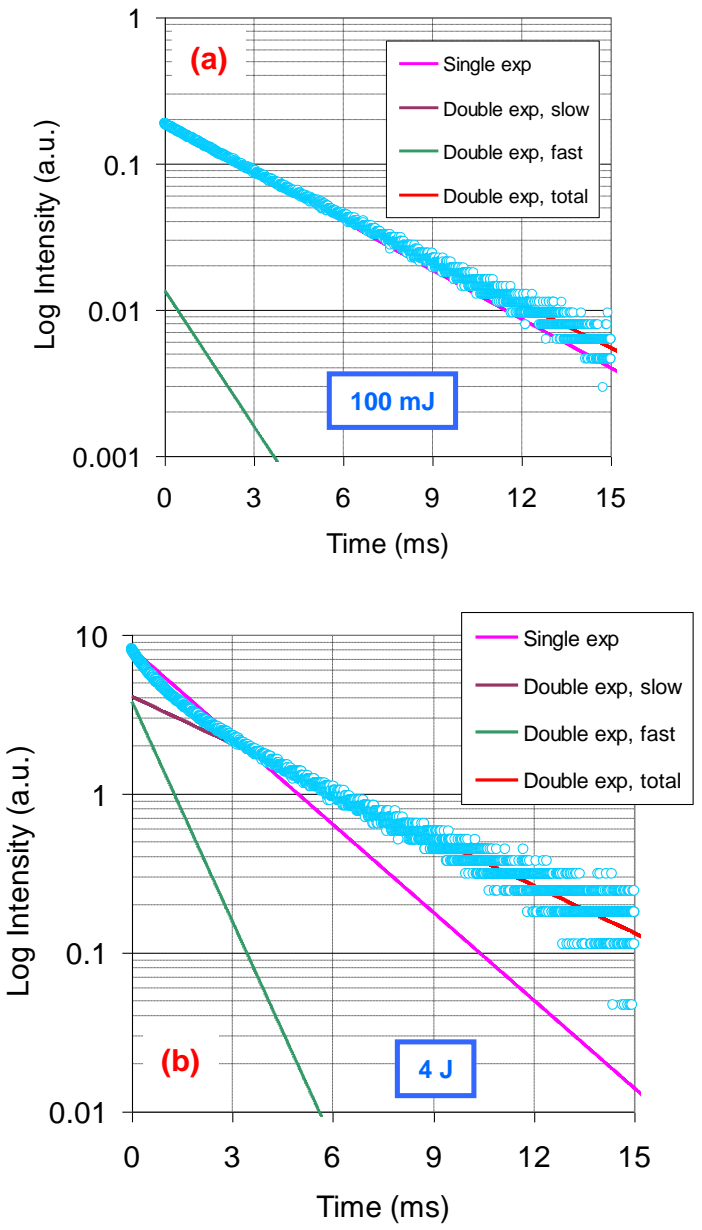

Figure 8: Sample double exponential fit to the measured roomtemperature experimental decay curves of the $25 \%$ doped YLF sample at an incident energy of (a) $100 \mathrm{~mJ}$ and (b) $4 \mathrm{~J}$. The data is taken using a $50 \mu \mathrm{m}$ pinhole aperture to minimize radiation trapping effects. The graph clearly shows the contribution of fast and slow component of double exponential fit. Single exponential fit curves are also included to for comparison. 
Data in Figs. 7 and 8 clearly show that, as the excitation intensity increases, or as the inversion level of the sample increases, a fast decay path appears in the measured emission decay curves. Moreover, the strength of this decay compared to the slow component increases with increasing excitation energy (Fig. 7 (b)). The rate of fast component also increases slowly with increasing excitation energy: has a value of around $2 \mathrm{~ms}$ at low intensities, which decreased to around 900 $\mu$ s level at high intensities. So we see that, with increasing inversion, the fast process gets faster and stronger. On the contrary, the slow component, which we attribute to the main transition of the laser level, do not get effected too much, and decays with a lifetime of around $4.5 \mathrm{~ms}$ at all intensity levels.
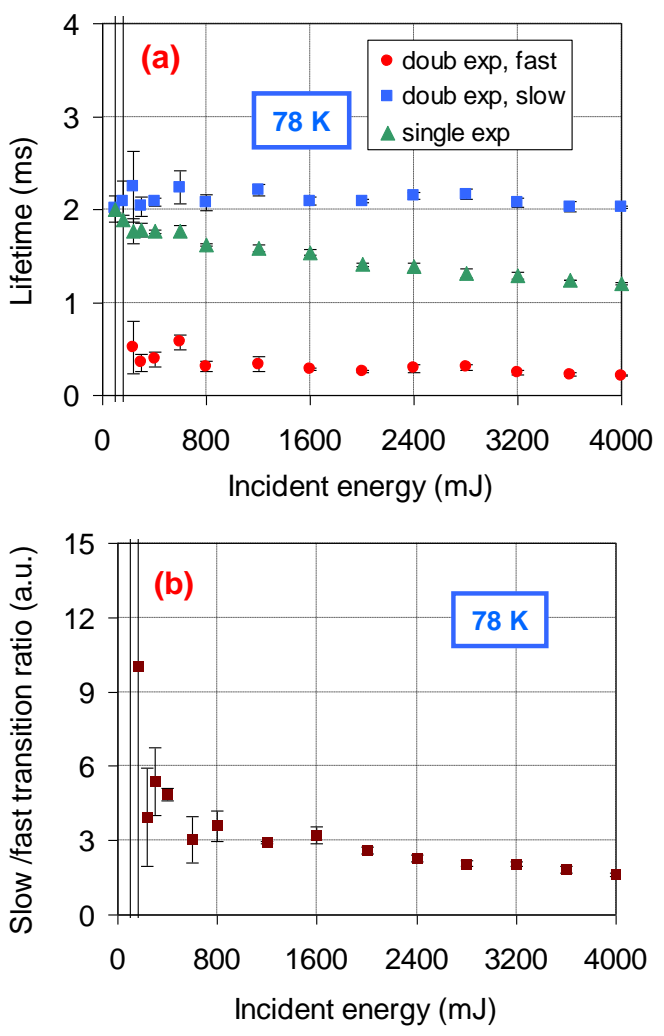

Figure 9: (a) Measured variation of fluorescence lifetime with incident pump excitation energy for the $25 \%$ Yb-doped YLF sample at $78 \mathrm{~K}$. The results are obtained via making a double exponential fit to the measured decay curves. (b) Corresponding variation of the ratio of slow and fast component strength of double exponential fit with temperature (A/B ratio in Eq. (2)).

A similar trend is observed for the $78 \mathrm{~K}$ data, where the main slow transition decayed with a lifetime of around 2-2.2 $\mathrm{ms}$ at all excitation intensity levels (Fig. 9). Comparing Fig. 7 (b) and Fig. 9 (b), we see that, the strength of the fast transition compared to the slow transition was also similar at 78 and 300 $\mathrm{K}$, and followed a similar change with excitation intensity. On the other hand, we have measured a much shorter fast component at $78 \mathrm{~K}$, which decayed within $500 \mu$ s at low intensities and within $200 \mu \mathrm{s}$ at high intensities. We believe that this is probably partially due to the increased absorption of the system at $78 \mathrm{~K}$ compared to $300 \mathrm{~K}$, enabling higher inversion levels. We also believe that, the fast initial decay of fluorescence signal is potentially due to (i) the redistribution of energy from the higher lying laser level of the $\mathrm{Yb}^{+3}$ ion $\left({ }^{2} \mathrm{~F}_{5 / 2}\right.$ level) into the other rare earth impurity ions via upconversion mechanism [55-57], and/or (ii) amplified spontaneous emission mechanism that becomes effective at very high inversion levels (gain increases at lower temperatures, which might also fasten the ASE process) [48, 49]. For our case, since the expected level of impurities are quite low (as it will be discussed in the next section), the ASE process is most probably the dominant mechanism in the observed behavior, which underlines the importance of detailed ASE analysis for successful design of Yb:YLF thindisk and slab lasers/amplifiers.

\subsection{Room-temperature upconversion spectrum of $25 \%$ Yb-doped YLF sample}

As mentioned earlier, we have observed strong upconversion from our $25 \% \mathrm{Yb}$-doped samples both at cryogenic and room temperatures. In this sub-section, we will present the spectra of observed emission in different spectral regions.

Fig. 10 and Fig. 11 show the measured emission spectra from the 25\% Yb-doped YLF sample at room temperature at several different excitation intensities in the $200-1100 \mathrm{~nm}$ and 1980-2100 nm spectral ranges, respectively. To start with, Fig. 10 (a) shows the strength of upconversion spectra compared to the main $1 \mu \mathrm{m}$ emission, where we clearly see that the emission in the main spectrum is much stronger (>>100-1000 times in strength) than the emission in the upconversion spectra. We have also seen that the strength of upconversion spectra roughly scales linearly with inversion (inversion level of the samples were estimated considering pump saturation effects).

Upconversion in Yb:YLF was already reported in $[17,32$, 45], and the observed emission spectrum were also identified and the underlying transitions were mostly recovered. Comparing our measurements with the published spectral data in literature, we have noticed that our $25 \% \mathrm{Yb}^{+3}$-doped YLF sample also possesses fingerprints of $\mathrm{Er}^{+3}, \mathrm{Ho}^{+3}$, and $\mathrm{Tm}^{+3}$ ions.

As an example, looking at the measured emission spectrum:

- the broad emission around $2050 \mathrm{~nm}$ (Fig. $11,{ }^{5} \mathrm{I}_{7}$ to ${ }^{5} \mathrm{I}_{8}$ decay) clearly indicates the presence of $\mathrm{Ho}^{+3}$ ion in our sample [58],

- the emission in the 740-830 nm range (Fig. 10 (f)) possesses fingerprints of $\mathrm{Tm}^{+3}$ ion $\left({ }^{3} \mathrm{H}_{4}\right.$ to ${ }^{3} \mathrm{H}_{6}$ decay) $[58,59]$,

- the emission peaks around $550 \mathrm{~nm}$ (Fig. 10 (d)) indicates the presence of $\mathrm{Er}^{+3}\left({ }^{4} \mathrm{~S}_{3 / 2}\right.$ to ${ }^{4} \mathrm{I}_{15 / 2}$ transition) [59], whereas the emission peak around $540 \mathrm{~nm}$ could be attributed to the ${ }^{5} \mathrm{~S}_{2}$ to ${ }^{5} \mathrm{I}_{8}$ transition of $\mathrm{Ho}^{+3}$ ion $[58,59]$,

- the blue emission band in the $450-500 \mathrm{~nm}$ spectral region (Fig. 10 (c)) is due to the ${ }^{1} \mathrm{G}_{4}$ to ${ }^{3} \mathrm{H}_{6}$ transition of the $\mathrm{Tm}^{+3}$ ion $[58,59]$,

- the emission spectrum near $650 \mathrm{~nm}$ is potentially due to the ${ }^{1} \mathrm{G}_{4}$ to ${ }^{3} \mathrm{~F}_{4}$ transition of $\mathrm{Tm}^{+3}$ ion $[58,59]$ and the ${ }^{4} \mathrm{~F}_{9 / 2}$ to ${ }^{4} \mathrm{I}_{15 / 2}$ transition of $\mathrm{Er}^{+3}$ ion [9], 
- the weak emission near $410 \mathrm{~nm}$ (Fig. 10 (b)) has been attributed to the ${ }^{2} \mathrm{H}_{9 / 2}$ to ${ }^{4} \mathrm{I}_{15 / 2}$ transition of $\mathrm{Er}^{+3}$ ion [32],

- the weak green emission around $500 \mathrm{~nm}$ might be due to $\mathrm{Yb}^{+3}$ pair emission [32].
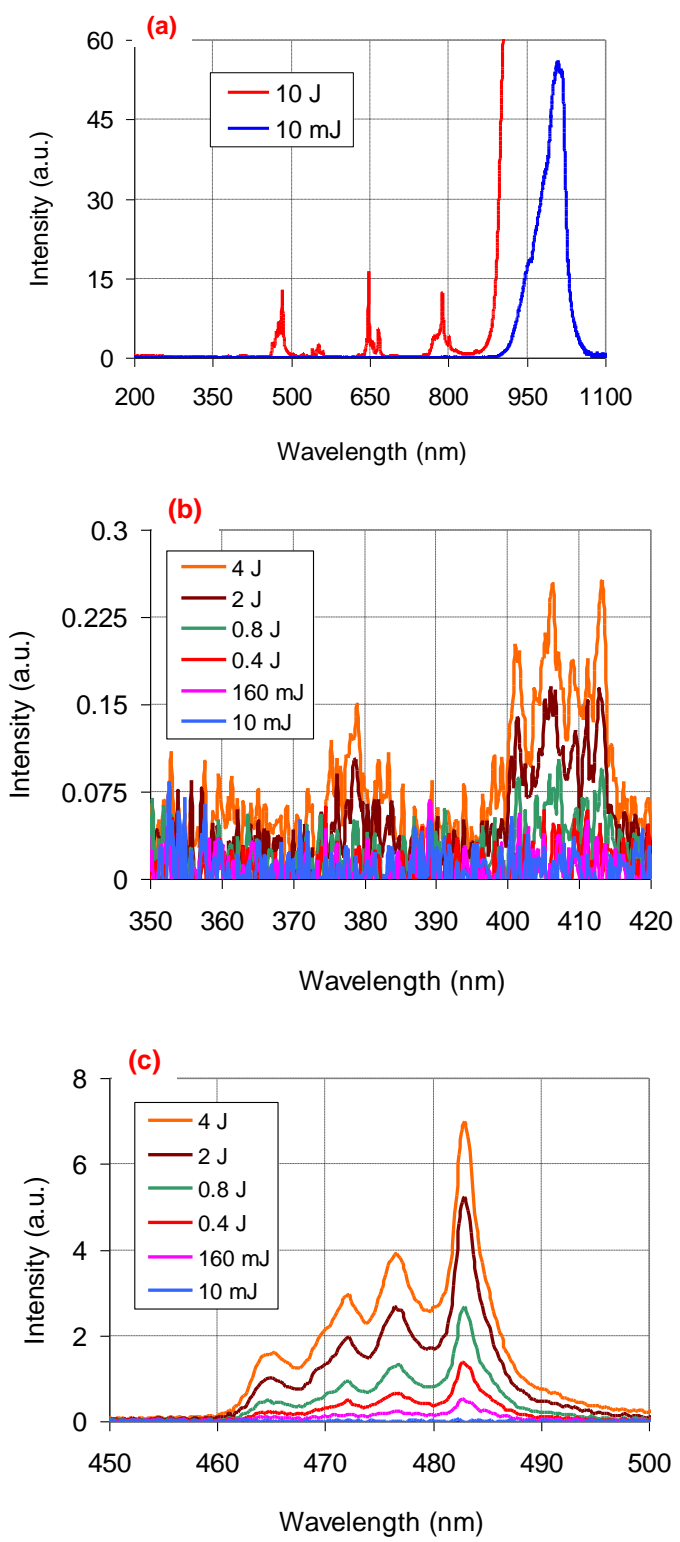

Figure 10: (a-f) Measured room-temperature emission and upconversion spectrum of $25 \% \mathrm{Yb}$-doped YLF sample at different pump excitation energies. (a) $200-1100 \mathrm{~nm}$, (b) $350-420 \mathrm{~nm}$, (c) $150-$ $500 \mathrm{~nm}$, (d) $500-575 \mathrm{~nm}$, (e) $620-680 \mathrm{~nm}$, (f) $740-830 \mathrm{~nm}$.

It is clear from the observed emission spectra that, energy transfer from the higher lying laser level of the $\mathrm{Yb}^{+3}$ ion $\left({ }^{2} \mathrm{~F}_{5 / 2}\right.$ level) into these impurity ions create loss of inversion and gain. Furthermore, besides the radiative decay lines we observe as upconversion emission, the energy levels of the $\mathrm{Tm}, \mathrm{Er}$ and $\mathrm{Tm}$ also possess non-radiative transitions, and via multi-phonon emission impurity ions could also create parasitic heating [60]. Hence, presence of rare-earth impurities in highly Yb-doped YLF samples might prevent efficient operation of lasers/amplifiers that intrinsically rely on highly-doped samples such as in thin-disk geometry.
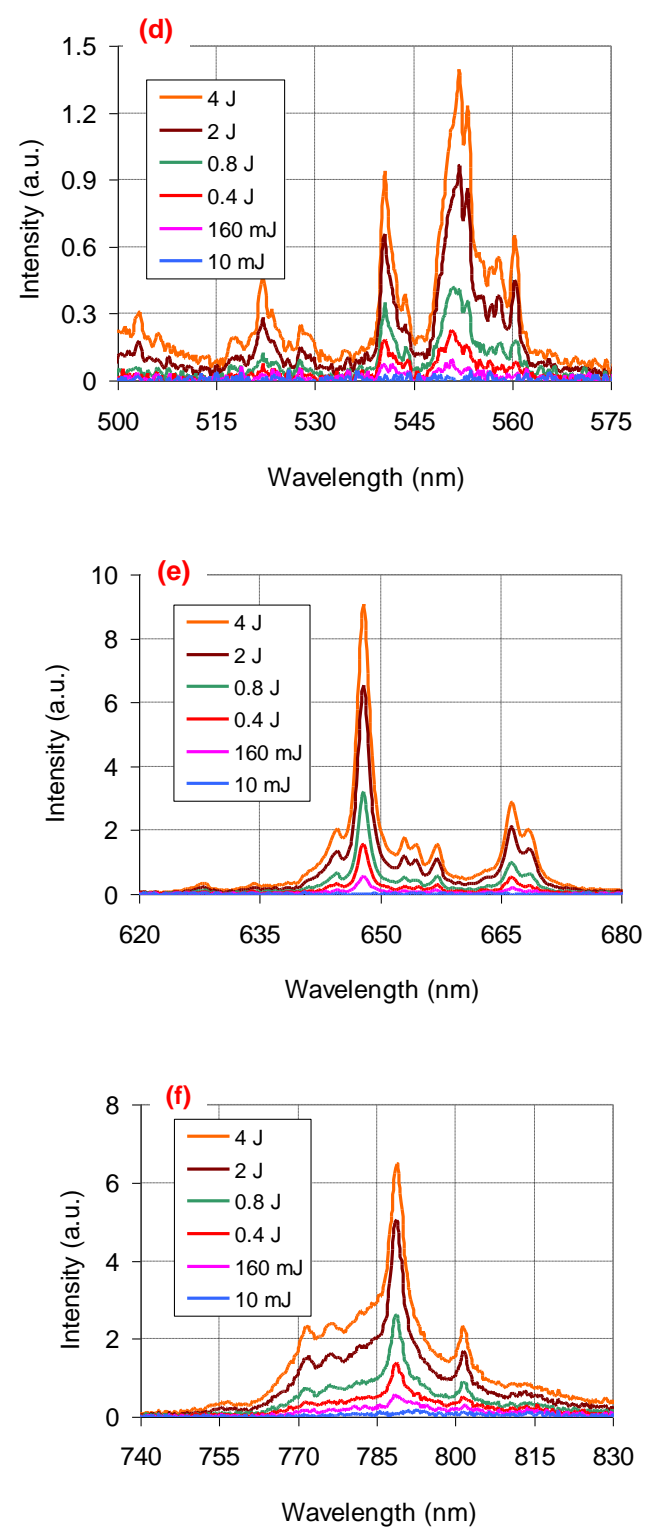

Figure 10: (a-f) Measured room-temperature emission and upconversion spectrum of $25 \%$ Yb-doped YLF sample at different pump excitation energies. (a) $200-1100 \mathrm{~nm}$, (b) $350-420 \mathrm{~nm}$, (c) $150-$ $500 \mathrm{~nm}$, (d) $500-575 \mathrm{~nm}$, (e) $620-680 \mathrm{~nm}$, (f) $740-830 \mathrm{~nm}$.

In closing we would like to mention that we have also observed very weak green emission from the $0.5 \%$ and $1 \%$ $\mathrm{Yb}$-doped samples. The emission was easily visible to the eye due to the spectral sensitivity of human eye in this spectral region, but a fast measurement with a spectrometer showed that the green emission strength is more than five orders of magnitude lower than the main $1 \mu \mathrm{m}$ emission. We believe some of this emission is due to $\mathrm{Yb}^{+3}$ ion pair emission [32], but we have also measured spectral peaks around 540 and 550 
$\mathrm{nm}$, indicating trace amounts of $\mathrm{Er}^{+3}$ and $\mathrm{Ho}^{+3}$ impurity even in these lowly doped samples.

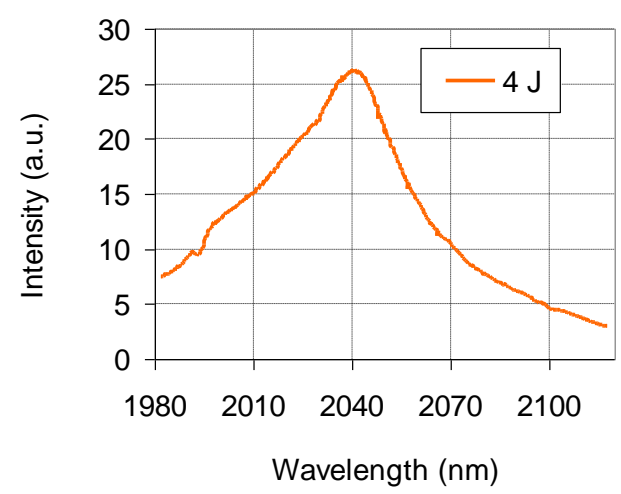

Figure 11: Measured room-temperature emission spectrum of $25 \%$ Yb-doped YLF sample around $2050 \mathrm{~nm}$.

\subsection{Timing dynamics of different colours of upconversion spectrum}
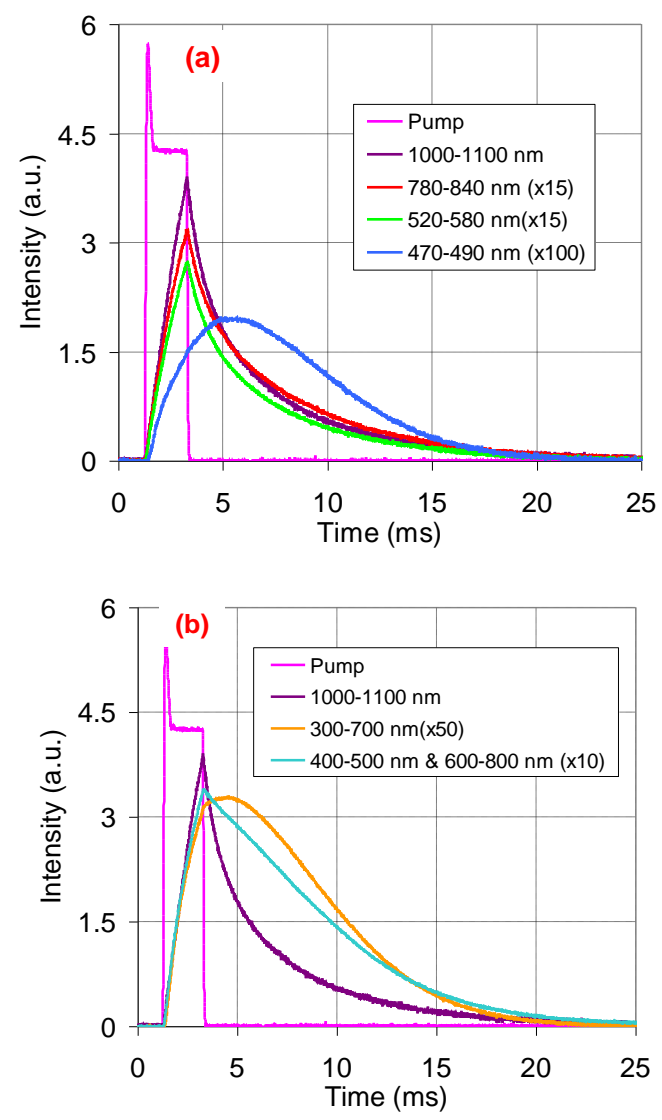

Figure 12: Measured emission time dynamics of $25 \% \mathrm{Yb}$-doped YLF sample at different spectral regions. The data is taken at an incident excitation energy of around $4 \mathrm{~J}$, and using a $500 \mu \mathrm{m}$ pinhole aperture to minimize radiation trapping effects. The $2 \mathrm{~ms}$ long pump excitation pulse (with a $2 \mathrm{~kW}$ peak power) is also shown.

In order to get a little more insight on the energy transfer process between the laser active $\mathrm{Yb}^{+3}$ ion and the other impurities, we have also characterized the decay dynamics of the different emission wavelengths in the upconversion spectra at room temperature. Fig. 12 shows the measured dynamics of emission at different wavelengths. As mentioned earlier, the emission in the main $1 \mu \mathrm{m}$ region is orders of magnitude stronger than upconversion emission, and hence in Fig. 12, the magnitude of emission decays at other colors is multiplied to scale them into levels close to the main line. Also, a relatively high excitation energy $(4 \mathrm{~J})$ is applied to the sample and a relatively large pinhole $(500 \mu \mathrm{m})$ was used to achieve enough signal to noise ratio for the upconversion signal decay lines. Hence, the effect of radiation trapping might be strong in the measurements shown in Fig. 12. As a final note, the intensities shown are in arbitrary units, since we did not correct for the spectral response of the $\mathrm{Si}$ photodiode, which has a better sensitivity at the upconversion wavelengths.

A close look at Fig. 12 suggests a few hints on the underlying processes. The excitation source (pump diode signal) is also shown in Fig. 12, and the end of the pump signal initiates a clean observation window for us. First of all, the emissions in the $520-580 \mathrm{~nm}$ and $780-820 \mathrm{~nm}$ range are mostly in phase with the main emission line in the 1000-1100 $\mathrm{nm}$ spectral region (the signal maximum is at the same position and also the decay curves show similar double exponential behavior). Best-fit results to the measured experimental decay curves are summarized in Table 1. For the decays in these 3 spectral regions, we have found a slow response time of around $5 \mathrm{~ms}$ and a fast response time of around $1 \mathrm{~ms}$ in all cases. Note that, a good fit to the measured data could be obtained for decay curves in this 3 spectral regions, whereas due to the non-exponential nature of the measured decays in the other spectral ranges, the fit to the experimental measured decay were not very good, and the values in Table 1 should be taken as representative decay constants.

\begin{tabular}{|c|c|c|c|c|}
\hline $\begin{array}{c}\text { Spectral } \\
\text { region } \\
(\mathrm{nm})\end{array}$ & $\begin{array}{c}\text { Single exp } \\
(\mathrm{ms})\end{array}$ & $\begin{array}{c}\text { Double exp } \\
\text { slow (ms) }\end{array}$ & $\begin{array}{c}\text { Double exp } \\
\text { fast (ms) }\end{array}$ & $\begin{array}{c}\text { Double exp } \\
\text { slow to fast } \\
\text { ratio (a.u.) }\end{array}$ \\
\hline $1000-1100$ & $2.96 \pm 0.04$ & $5.15 \pm 0.1$ & $0.97 \pm 0.03$ & $1.32 \pm 0.03$ \\
\hline $520-580$ & $3.40 \pm 0.06$ & $4.87 \pm 0.06$ & $0.90 \pm 0.03$ & $2.18 \pm 0.04$ \\
\hline $780-840$ & $3.90 \pm 0.06$ & $5.25 \pm 0.15$ & $0.74 \pm 0.04$ & $2.91 \pm 0.12$ \\
\hline $470-490$ & $6.51 \pm 0.15$ & - & - & - \\
\hline $300-700$ & $6.73 \pm 0.18$ & - & & - \\
\hline $\begin{array}{c}400-500 \& \\
600-900\end{array}$ & $6.81 \pm 0.03$ & - & - & - \\
\hline
\end{tabular}

Table 1: Summary of single and double exponential fit results to the emission decay curves in different spectral regions.

As an example, note that, the peak of the emission signal at 470-490 and 300-700 $\mathrm{nm}$ spectral range lags behind the main emission (not in phase). The lag time is around $1 \mathrm{~ms}$ for the $300-700 \mathrm{~nm}$ band and as large as $2.5 \mathrm{~ms}$ for the $470-490$ $\mathrm{nm}$ transition (a simple single exponential fit gives the rise time of the energy transfer as $1.25 \mathrm{~ms}$ ). Emission in the blue region (450-500 $\mathrm{nm}$ ) is believed to originate from the ${ }^{1} \mathrm{G}_{4}$ to ${ }^{3} \mathrm{H}_{6}$ transition of the $\mathrm{Tm}^{+3}$ ion $[58,59]$. Matos et al. explains several possible pathways of energy transfer from the $\mathrm{Yb}$ ion to the Tm ion [61], including energy-transfer upconversion, 
cross relaxation and even a back transfer process (see Fig. 1 in [61]). A Yb-Tm energy transfer process rise-time of around $800 \mu \mathrm{s}$ was reported in [61], that is slightly shorter than our measurement $(1.2 \mathrm{~ms})$. On the other hand, while analyzing the influence of rare earth impurities in refrigeration efficiency in Yb:YLF, Di Lieto et al. proposed another path [17], the energy transfer from the $\mathrm{Yb}^{+3}$ ions to $\mathrm{Tm}^{+3}$ is probably not direct, and occurs via the $\mathrm{Er}^{+3}$ impurity (energy transfers first from $\mathrm{Yb}$ to Er and then from Er to Tm, see Fig. 6 in [17]). The process involves emission of Erbium ion from the ${ }^{4} \mathbf{I}_{13 / 2}$ energy level, which has a long lifetime of $15 \mathrm{~ms}$ [62]. Hence, the relatively long delay $(2.5 \mathrm{~ms})$ we have measured for the 470 $490 \mathrm{~nm}$ emission could be potentially explained by this model [17]. This simple example shows that, combined with the effect of radiation trapping that is already strongly present in the highly $\mathrm{Yb}$-doped sample, the overall timing of energy transfer process gets quite complex in the highly Yb-doped YLF sample with Tm, Er and Ho impurities [9].

\section{Conclusion}

In conclusion, we have presented a detailed study focused on variation of fluorescence lifetime of Yb:YLF with temperature and doping. The data with the lowly doped (0.5$1 \%)$ samples using the pinhole method confirmed the earlier literature: fluorescence lifetime is measured to be $1.97 \mathrm{~ms}$ and $2.1 \mathrm{~ms}$ at $78 \mathrm{~K}$ and $300 \mathrm{~K}$, respectively. On the other hand, we have observed a double exponential behavior and excitation intensity dependent decay process in the highlydoped $(25 \%)$ YLF sample, which is to our knowledge, was not reported earlier. We believe that this double-exponential behavior is mostly due to the amplified spontaneous emission process, and our data shows that suppression of ASE will be a challenge in the design of thin-disk Yb:YLF lasers/amplifiers especially for those operating at cryogenic temperatures. We have further provided detailed upconversion spectrum and initial measurements revealing some insights into the energy transfer process between the $\mathrm{Yb}$ ion and the other impurities.

It is clear that, more work is required to understand whether the Yb:YLF crystal growth process could be optimized to minimize the impurities in highly Yb-doped samples. Future work is also required to see the importance of this impurities and parasitic energy transfer process on laser/amplifier performance. We hope the initial findings presented in this work to be useful to laser engineers and scientists working with Yb:YLF systems.

\section{Acknowledgements}

UD acknowledges support from BAGEP Award of the Bilim Akademisi. The authors acknowledge support from previous group members L. E. Zapata, K. Zapata for establishing the indium-bonding technology for YLF at CFEL-DESY.

\section{Disclosures}

The authors declare no conflicts of interest.
1. J. Kawanaka, H. Nishioka, N. Inoue, and K. Ueda, "Tunable continuouswave $\mathrm{Yb}$ : YLF laser operation with a diode-pumped chirped-pulse amplification system," Applied Optics 40, 3542-3546 (2001).

2. J. Kawanaka, S. Tokita, H. Nishioka, M. Fujita, T. Kawashima, T. Yanagitani, K. Ueda, and Y. Izawa, "Cryogenically-cooled active-mirror oscillator with $\mathrm{Yb}$-doped material for inertial fusion energy driver, " in Pacific Rim Conference on Lasers \& Electro-Optics(IEEE, Tokyo, Japan, 2005).

3. K. Beil, S. T. Fredrich-Thornton, C. Kränkel, K. Petermann, D. Parisi, M. Tonelli, and G. Huber, "New thin disk laser materials: Yb:ScYLO and Yb:YLF," in Conference on Lasers and Electro-Optics Europe(IEEE, Munich, Germany, 2011).

4. M. Vannini, G. Toci, D. Alderighi, D. Parisi, F. Cornacchia, and M. Tonelli, "High efficiency room temperature laser emission in heavily doped Yb : YLF," Optics Express 15, 7994-8002 (2007).

5. D. Alderighi, A. Pirri, G. Toci, and M. Vannini, "Tunability enhancement of Yb:YLF based laser," Optics Express 18, 2236-2241 (2010).

6. N. Coluccelli, G. Galzerano, L. Bonelli, A. Di Lieto, M. Tonelli, and P. Laporta, "Diode-pumped passively mode-locked Yb : YLF laser," Optics Express 16, 2922-2927 (2008).

7. F. Pirzio, L. Fregnani, A. Volpi, A. Di Lieto, M. Tonelli, and A. Agnesi, "87 fs pulse generation in a diode-pumped semiconductor saturable absorber mirror mode-locked Yb:YLF laser," Applied Optics 55, 4414-4417 (2016).

8. W. Bolanos, F. Starecki, A. Braud, J. L. Doualan, R. Moncorge, and P. Camy, "2.8 W end-pumped Yb3+:LiYF4 waveguide laser," Optics Letters 38, 5377-5380 (2013).

9. A. Bensalah, Y. Guyot, A. Brenier, H. Sato, T. Fukuda, and G. Boulon, "Spectroscopic properties of Yb3+: LuLiF4 crystal grown by the Czochralski method for laser applications and evaluation of quenching processes: a comparison with Yb3+: YLiF4," Journal of Alloys and Compounds 380, 15-26 (2004).

10. M. Eichhorn, "Quasi-three-level solid-state lasers in the near and mid infrared based on trivalent rare earth ions," Applied Physics B-Lasers and Optics 93, 269-316 (2008).

11. U. Demirbas, J. Thesinga, M. Kellert, F. X. Kärtner, and M. Pergament, "Comparison of different in situ optical temperature probing techniques for cryogenic Yb:YLF," Optical Materials Express 10, 3403-3413 (2020).

12. S. Melgaard, "Cryogenic optical refrigeration: Laser cooling of solids below 123 K," (University of New Mexico, 2013).

13. S. D. Melgaard, A. R. Albrecht, M. P. Hehlen, and M. Sheik-Bahae, "Solid-state optical refrigeration to sub-100 Kelvin regime," Scientific Reports 6 (2016).

14. D. V. Seletskiy, S. D. Melgaard, S. Bigotta, A. Di Lieto, M. Tonelli, and M. Sheik-Bahae, "Laser cooling of solids to cryogenic temperatures," Nature Photonics 4, 161-164 (2010).

15. S. Melgaard, D. Seletskiy, V. Polyak, Y. Asmerom, and M. Sheik-Bahae, "Identification of parasitic losses in Yb:YLF and prospects for optical refrigeration down to 80K," Optics Express 22, 7756-7764 (2014).

16. A. Pant, X. J. Xia, E. J. Davis, and P. J. Pauzauskie, "Solid-state laser refrigeration of a composite semiconductor Yb:YLiF4 optomechanical resonator," Nature Communications 11 (2020).

17. A. Di Lieto, A. Sottile, A. Volpi, Z. H. Zhang, D. V. Seletskiy, and M. Tonelli, "Influence of other rare earth ions on the optical refrigeration efficiency in Yb:YLF crystals," Optics Express 22, 28572-28583 (2014).

18. A. Volpi, A. Di Lieto, and M. Tonelli, "Novel approach for solid state cryocoolers," Optics Express 23, 8216-8226 (2015).

19. J. Kawanaka, S. Tokita, H. Nishioka, M. Fujita, K. Yamakawa, K. Ueda, and Y. Izawa, "Dramatically improved laser characteristics of diode-pumped Yb-doped materials at low temperature," Laser Physics 15, 1306-1312 (2005).

20. J. Kawanaka, K. Yamakawa, H. Nishioka, and K. Ueda, "Improved highfield laser characteristics of a diode-pumped Yb : LiYF4 crystal at low temperature," Optics Express 10, 455-460 (2002).

21. R. L. Aggarwal, D. J. Ripin, J. R. Ochoa, and T. Y. Fan, "Measurement of thermo-optic properties of Y3AI5O12, Lu3AI5O12, YAIO(3), LiYF4, LiLuF4, BaY2F8, KGd(WO4)(2), and $\mathrm{KY}(\mathrm{WO} 4)(2)$ laser crystals in the $80-300 \mathrm{~K}$ temperature range," Journal of Applied Physics 98 (2005).

22. L. E. Zapata, D. J. Ripin, and T. Y. Fan, "Power scaling of cryogenic Yb:LiYF4 lasers," Optics Letters 35, 1854-1856 (2010).

\section{References}


23. D. Rand, D. Miller, D. J. Ripin, and T. Y. Fan, "Cryogenic Yb3+-doped materials for pulsed solid-state laser applications [Invited]," Optical Materials Express 1, 434-450 (2011).

24. D. E. Miller, L. E. Zapata, D. J. Ripin, and T. Y. Fan, "Sub-picosecond pulses at $100 \mathrm{~W}$ average power from a Yb:YLF chirped-pulse amplification system," Optics Letters 37, 2700-2702 (2012).

25. D. E. Miller, J. R. Ochoa, and T. Y. Fan, "Cryogenically cooled, 149 W, Q-switched, Yb:LiYF4 laser," Optics Letters 38, 4260-4261 (2013).

26. U. Demirbas, H. Cankaya, J. Thesinga, F. X. Kartner, and M. Pergament, "Efficient, diode-pumped, high-power (>300W) cryogenic $\mathrm{Yb}: Y L F$ laser with broad-tunability (995-1020.5 nm): investigation of E//aaxis for lasing " Optics Express 27, 36562-36579 (2019).

27. U. Demirbas, H. Cankaya, Y. Hua, J. Thesinga, M. Pergament, and F. X. Kaertner, "20-mJ, sub-ps pulses at up to $70 \mathrm{~W}$ average power from a cryogenic Yb:YLF regenerative amplifier " Optics Express 28, 2466-2479 (2020).

28. U. Demirbas, J. Thesinga, H. Cankaya, M. Kellert, F. X. Kartner, and M. Pergament, "High-power passively mode-locked cryogenic Yb:YLF laser," Optics Letters 45, 2050-2053 (2020).

29. H. Cankaya, U. Demirbas, Y. Hua, M. Hemmer, L. E. Zapata, M. Pergament, and F. X. Kärtner, "190-mJ Cryogenically-Cooled Yb:YLF Amplifier System at $1019.7 \mathrm{~nm}, "$ OSA continuum 2, 3547-3553 (2019).

30. L. D. DeLoach, S. A. Payne, L. L. Chase, L. K. Smith, W. L. Kway, and W. F. Krupke, "Evaluation of Absorption and Emission Properties of $\mathrm{Yb}$ Doped Crystals for Laser Applications," IEEE Journal of Quantum Electronics 29, 1179-1191 (1993).

31. T. Y. Fan, D. J. Ripin, R. L. Aggarwal, J. R. Ochoa, B. Chann, M. Tilleman, and J. Spitzberg, "Cryogenic Yb3+-doped solid-state lasers," leee Journal of Selected Topics in Quantum Electronics 13, 448-459 (2007).

32. G. Boulon, Y. Guyot, M. Ito, A. Bensalah, C. Goutaudier, G. Panczer, and J. C. Gacon, "From optical spectroscopy to a concentration quenching model and a theoretical approach to laser optimization for Yb3+-doped YLiF4 crystals," Molecular Physics 102, 1119-1132 (2004).

33. E. A. Milne, "The diffusion of imprisoned radiation through a gas," J. Lond. Math. Soc. 1, 40-51 (1926).

34. M. Yokota, and O. Tanimoto, "Effects of Diffusion on Energy Transfer by Resonance," Journal of the Physical Society of Japan 22, 779-784 (1967).

35. M. J. Weber, "Luminescence Decay by Energy Migration and Transfer: Observation of Diffusion-Limited Relaxation," Physical Review B 4, 29322939 (1971).

36. F. Auzel, F. Bonfigli, S. Gagliari, and G. Baldacchini, "The interplay of self-trapping and self-quenching for resonant transitions in solids; role of a cavity," Journal of Luminescence 94, 293-297 (2001).

37. F. Auzel, G. Baldacchini, L. Laversenne, and G. Boulon, "Radiation trapping and self-quenching analysis in $\mathrm{Yb3}+, \mathrm{Er} 3+$, and $\mathrm{Ho} 3+$ doped $\mathrm{Y} 2 \mathrm{O} 3, "$ Optical Materials 24, 103-109 (2003).

38. A. Brenier, "Excited-state dynamics including radiative diffusion in quasi-three-level laser crystals: application to Yb3+-doped Y3AI5O12," Journal of the Optical Society of America B-Optical Physics 23, 2209-2216 (2006).

39. S. Guy, "Modelization of lifetime measurement in the presence of radiation trapping in solid-state materials," Physical Review B 73 (2006).

40. M. Eichhorn, "Fluorescence reabsorption and its effects on the local effective excitation lifetime," Applied Physics B-Lasers and Optics 96, 369377 (2009)

41. A. Bensalah, M. Ito, Y. Guyot, C. Goutaudier, A. Jouini, A. Brenier, H. Sato, T. Fukuda, and G. Boulon, "Spectroscopic properties and quenching processes of $\mathrm{Yb3}+$ in fluoride single crystals for laser applications," Journal of Luminescence 122, 444-446 (2007).

42. F. D. Patel, E. C. Honea, J. Speth, S. A. Payne, R. Hutcheson, and R. Equall, "Laser demonstration of Yb3Al5O12 (YbAG) and materials properties of highly doped Yb : YAG," leee Journal of Quantum Electronics 37, 135-144 (2001).

43. T. Y. Fan, "Heat Generation in Nd:YAG and Yb:YAG," IEEE Journal of Quantum Electronics 29, 1457-1459 (1993).

44. D. L. Dexter, "A Theory of Sensitized Luminescence in Solids," Journal of Chemical Physics 21, 836-850 (1953).
45. A. Volpi, G. Cittadino, A. Lieto, A. Cassanho, H. P. Jenssen, and M. Tonelli, "Investigation of Yb-doped LiLuF4 single crystals for optical cooling," Optical Engineering 56 (2017).

46. M. Smrz, T. Miura, M. Chyla, S. Nagisetty, O. Novak, A. Endo, and T. Mocek, "Suppression of nonlinear phonon relaxation in Yb:YAG thin disk via zero phonon line pumping," Optics Letters 39, 4919-4922 (2014).

47. F. Carl, L. Birk, B. Grauel, M. Pons, C. Würth, U. Resch-Genger, and M Haase, "LiYF4:Yb/LiYF4 and LiYF4:Yb,Er/LiYF4 core/shell nanocrystals with luminescence decay times similar to YLF laser crystals and the upconversion quantum yield of the $\mathrm{Yb}$,Er doped nanocrystals," Nano Research (2020).

48. A. Antognini, K. Schuhmann, F. D. Amaro, F. Biraben, A. Dax, A. Giesen, T. Graf, T. W. Hansch, P. Indelicato, L. Julien, C. Y. Kao, P. E. Knowles, F. Kottmann, E. Le Bigot, Y. W. Liu, L. Ludhova, N. Moschuring, F. Mulhauser, T. Nebel, F. Nez, P. Rabinowitz, C. Schwob, D. Taqqu, and R. Pohl, "Thin-Disk Yb:YAG Oscillator-Amplifier Laser, ASE, and Effective Yb:YAG Lifetime," leee Journal of Quantum Electronics 45, 983-995 (2009).

49. P. Peterson, A. Gavrielides, T. C. Newell, N. Vretenar, and W. P. Latham, "ASE in thin disk lasers: theory and experiment," Optics Express 19, 25672-25684 (2011).

50. J. Korner, M. Kruger, J. Reiter, A. Munzer, J. Hein, and M. C. Kaluza, "Temperature dependent spectroscopic study of $\mathrm{Yb}^{+3}$-doped $\mathrm{KG}\left(\mathrm{WO}_{4}\right)_{2}$, $\mathrm{KY}\left(\mathrm{WO}_{4}\right)_{2}, \mathrm{YAlO}_{3}$ and $\mathrm{YliF}_{4}$ for laser applications," Optical Materials Express 10, 2425-2438 (2020).

51. H. Burton, C. Debardelaben, W. Amir, and T. A. Planchon, "Temperature dependence of Ti:Sapphire fluorescence spectra for the design of cryogenic cooled Ti:Sapphire CPA laser," Optics Express 25, 69546962 (2017).

52. G. Toci, "Lifetime measurements with the pinhole method in presence of radiation trapping: I-theoretical model," Applied Physics BLasers and Optics 106, 63-71 (2012).

53. G. Toci, D. Alderighi, A. Pirri, and M. Vannini, "Lifetime measurements with the pinhole method in presence of radiation trapping: II-application to Yb3+ doped ceramics and crystals," Applied Physics B-Lasers and Optics 106, 73-79 (2012).

54. H. Kuhn, S. T. Fredrich-Thornton, C. Krankel, R. Peters, and K. Petermann, "Model for the calculation of radiation trapping and description of the pinhole method," Optics Letters 32, 1908-1910 (2007).

55. F. Qin, H. Zhao, M. Y. Lv, Y. Zhou, L. P. Li, Z. J. Wang, Y. D. Zheng, Z. G. Zhang, and W. W. Cao, "Eliminating the "decoupling" effect of fluorescence intensity ratio thermometry for an upconversion pumped system," Journal of Luminescence 192, 184-187 (2017).

56. X. J. Pei, Y. B. Hou, S. L. Zhao, Z. Xu, and F. Teng, "Frequency upconversion of $\mathrm{Tm} 3+^{+}$and $\mathrm{Yb3}+$ codoped YLiF4 synthesized by hydrothermal method," Materials Chemistry and Physics 90, 270-274 (2005).

57. M. S. Liao, L. Wen, H. Y. Zhao, Y. Z. Fang, H. T. Sun, and L. L. Hu, "Mechanisms of $\mathrm{Yb3}+$ sensitization to $\mathrm{Tm} 3+$ for blue upconversion luminescence in fluorophosphate glass," Materials Letters 61, 470-472 (2007).

58. B. M. Walsh, N. P. Barnes, and B. Di Bartolo, "Branching ratios, cross sections, and radiative lifetimes of rare earth ions in solids: Application to Tm3+ and Ho3+ ions in LiYF4," Journal of Applied Physics 83, 2772-2787 (1998).

59. G. Cittadino, A. Volpi, A. Di Lieto, and M. Tonelli, "Co-doping of LiYF4 crystal: a virtuous effect of cooling efficiency," Journal of Physics D-Applied Physics 51 (2018).

60. X. Xia, A. Pant, A. S. Ganas, F. Jelezko, and P. J. Pauzauskie, "Quantum Point Defects for Solid-State Laser Refrigeration," Advanced Materials (2020).

61. P. S. F. De Matos, N. U. Wetter, L. Gomes, I. M. Ranieri, and S. L. Baldochi, "A high power $2.3 \mathrm{mu} \mathrm{m} \mathrm{Yb}: \mathrm{Tm}$ : YLF laser diode-pumped simultaneously at 685 and $960 \mathrm{~nm}$," Journal of Optics a-Pure and Applied Optics 10 (2008).

62. N. Ter-Gabrielyan, and V. Fromzel, "Cascade generation at 1.62, 1.73 and $2.8 \mathrm{mu} \mathrm{m}$ in the Er:YLF Q-switched laser," Optics Express 27, 2019920210 (2019) 